\title{
The ovine jugular vein as a model for interventional radiology procedures
}

\author{
Wei Lu' ${ }^{1,2}$, Won Kyu Park ${ }^{1,3}$, Barry Uchida ${ }^{1}$, Hans A. Timmermans ${ }^{1}$, \\ Dusan Pavcnik ${ }^{1}$, Frederick S. Keller ${ }^{1}$, Josef Rösch ${ }^{1}$
}
${ }^{1}$ Dotter Interventional Institute, Oregon Health Sciences University, 3181 SW Sam Jackson Park Road, L-342, Portland, OR 97239-3098, ²Department of Interventional Radiology, Nanfang Hospital, Southern Medial University, Guangzhou, Guangdong 510515, China, ${ }^{3}$ Department of Radiology, College of Medicine, Yeungnam University, 317-1 Daemyung-dong, Nam-gu, Daegu, 705-717, Korea

\begin{abstract}
Background. Detailed knowledge of the ovine jugular vein anatomy and physiology is a prerequisite for proper use of sheep as teaching or an experimental model in interventional radiology.

Material and methods. Ascending and descending jugular venograms in tilted position were done in 25 sheep to evaluate the jugular vein (JV) size and anatomy of its valves.

Results. The average maximal diameter of $50 \mathrm{JVs}$ was $13.34 \pm 1.18 \mathrm{~mm}$. Each vein contained an average of $4.36 \pm 0.98$ valves. All valves were competent and $96.3 \%$ were bicuspid.

Conclusions. Because of similarities between ovine JV and human femoral vein in regards to diameters, number and type of valves and function of their valves with increased central and hydrostatic pressure, the ovine JV is a good model for evaluation of creation of JV valve incompetence, percutaneous valve transplantation and evaluation of prosthetic valve devices.
\end{abstract}

Key words: jugular vein; experimental model, ovine; interventional radiology

\section{Introduction}

Percutaneous techniques have emerged as minimally invasive options in the treatment of chronic venous insufficiency. For replacement of diseased or absent venous valves,

Received 29 February 2008

Accepted 12 March 2008

Correspondence to: Dusan Pavcnik, M.D., Ph.D., Dotter Interventional Institute, Oregon Health Sciences University, 3181 SW Sam Jackson Park Road, L-342, Portland, OR 97239-3098, U.S.A.; Phone: +1 503494 3669; Fax: +1 503494 4258; Email: pavcnikd@ohsu.edu several artificial percutaneously implanted valves have been developed over the last 10 years. ${ }^{1-5}$ The ovine jugular vein (JV) has been often used for testing of the new valve devices because of its similar size to human femoral vein. 3,5-10 However, to our knowledge, there has not been a detailed study on the ovine JV angiographic anatomy, particularly regarding the number, distribution and type of its valves. The purpose of this study is to describe the angiographic anatomy of the ovine JV and its valves as a suitable model for interventional radiology procedures. 


\section{Materials and methods}

The study involved 25 adult female sheep weighing 53-74 kg (mean $64 \mathrm{~kg}$ ) and was a part of the following studies: testing a new bioprosthetic valve testing (7 sheep), attempts of creation of primary venous insufficiency ( 8 sheep) and testing new IVC filters (10 sheep). The Institutional Animal Care and Use Committee of Oregon Health \& Science University approved the protocols of these studies.

Animals fasted overnight with water available and were tranquilized intravenously with $7.5-10 \mathrm{mg}(0.05 \mathrm{mg} / \mathrm{lb})$ of Diazepam (Midazolam; Ben Venue Labs, Bedford, OH) and 400-800 mg (2.0 mg/lb) of Ketamine (Ketaset; Ft. Dodge Animal Health, Ft. Dodge, IA). Animals were then intubated. Inhalation anesthesia was maintained with 2-2.5\% Isoflurane (IsoFlo; Abbott Laboratories, Chicago, IL) and $2 \mathrm{~L} / \mathrm{min}$ of oxygen. To reduce salivation, $5 \mathrm{mg}$ Atropine sulfate (American Regent Laboratories, Shirley, NY) was administered intravenously. Antibiotics (10 mg/kg cefazolin) were given intramuscularly as single dose at the beginning of procedures. Respiratory rhythm and carbon dioxide saturation were monitored during procedure. A GE/OEC 9800 cardiac mobile system with digital imaging (GE Medical Systems/OEC, Salt Lake City, UT) was used for imaging.

Both JVs were percutaneously entered just below the jaw and $7 \mathrm{~cm}$ long $6.0 \mathrm{FR}$ Check-Flo vascular sheaths (Cook Medical, Bloomington, IN) were introduced and used to obtain venograms. A graduate measuring 0.035-inch wire guide (Cook Medical) was introduced into each sheath for calibration during venography. The right femoral vein was percutaneously entered and a $110 \mathrm{~cm}$ long 5F H1 Torcon Advantage catheter (Cook Medical) was introduced and advanced into the JV below its most central valve for descending venograms. Both ascending and descending venograms were performed with the sheep in approximately 30 degrees tilted position (head down) using hand injections of $10-20 \mathrm{ml}$ of contrast medium. Filming of each vein was performed in two projections and was prolonged to visualize the residual contrast in the valvular cusps. Simultaneous venograms of both JVs were also performed in anterio-posterior projection for visualization their anatomical relation. In 10 animals evaluated for IVC filters placement, the descending venograms of each JV valve were performed after the $\mathrm{H} 1$ catheter was passed through the competent central valves.

After venographic study of the JV anatomy, the animals underwent further testing according to the protocols. Four animals were terminated immediately after these studies and specimens of their JVs were obtained for comparison with their venograms. The other 21 animals were used for long-term evaluation.

The diameters of JVs were measured on the venograms and the number of venous valves, the type of valves (number of their cusps), and their distribution were carefully studied. The JVs were divided into thirds, the peripheral (distal), the middle and the central (proximal) segments.

\section{Results}

On the tilted ascending venograms, the JVs were well filled, distended and circular in shape. Their filling extended peripherally above the access site to the most peripheral competent valve. Some venous tributaries were also filled to their first venous valve. The JV diameters ranged from $9.8 \mathrm{~mm}$ to $15.2 \mathrm{~mm}$ with an average of $13.34 \pm 1.18$ $\mathrm{mm}$. The JV diameters in the peripheral segment ranged from $12.5 \mathrm{~mm}$ to 15.2 $\mathrm{mm}$ with an average of $14.28 \pm 1.06 \mathrm{~mm}$. In the middle segment vein diameters ranged 
Table 1. Jugular vein diameters, valve distribution and frequency

\begin{tabular}{cccc}
\hline Segments & Diameter $(\mathrm{mm})$ & Valve Distribution & Valve Frequency \\
\hline Peripheral & $14.28 \pm 1.06$ & $58(26.6 \%)$ & $92 \%$ \\
Middle & $12.68 \pm 1.14$ & $41(18.8 \%)$ & $76 \%$ \\
Central & $12.92 \pm 1.03$ & $119(54.6 \%)$ & $100 \%$ \\
\hline
\end{tabular}

from $10.6 \mathrm{~mm}$ to $13.7 \mathrm{~mm}$ with an average of $12.68 \pm 1.14 \mathrm{~mm}$. The JV diameters in the central third ranged from $9.8 \mathrm{~mm}$ to $13.2 \mathrm{~mm}$ with an average of $12.92 \pm 1.03$ $\mathrm{mm}$ (Table 1). The distended venograms often displayed the venous valves as faint linear defects inside sinuses, extending from the wall into lumen (Figure 1b). In the later phase of venograms valves were better visualized as their cusps contained some residual of contrast material and it was possible to define the number of cusps as well (Figures 1a, 1c). Altogether 218 valves were found in $50 \mathrm{JVs}$, with a range from 3-7 $(4.36 \pm 0.98)$ valves in each JV. Most valves (210) were bicuspid (96.3\%). Five valves had one cusp (monocusp - 2.3\%) and three had three cusps (tricuspid - 1.4\%). The major- ity of valves 119 (54.6\%) were distributed in the central venous segment that always contained at least two valves. In the middle segment, there were 41 (18.8\%) valves with 3 JVs containing two valves. Twelve middle segments were without valves. In the peripheral segment, there were 58 (26.6\%) valves. Four JVs peripheral segments were without valves.

The descending venograms displayed the valves and their types, whether they had one, two or three cusps well (Figure 2). The valves were competent and the hand injections filled the venous branches central to the valve. The axillary veins were always filled during injection below the central positioned valves. A perforating vein between the jugular and vertebral vein was filled
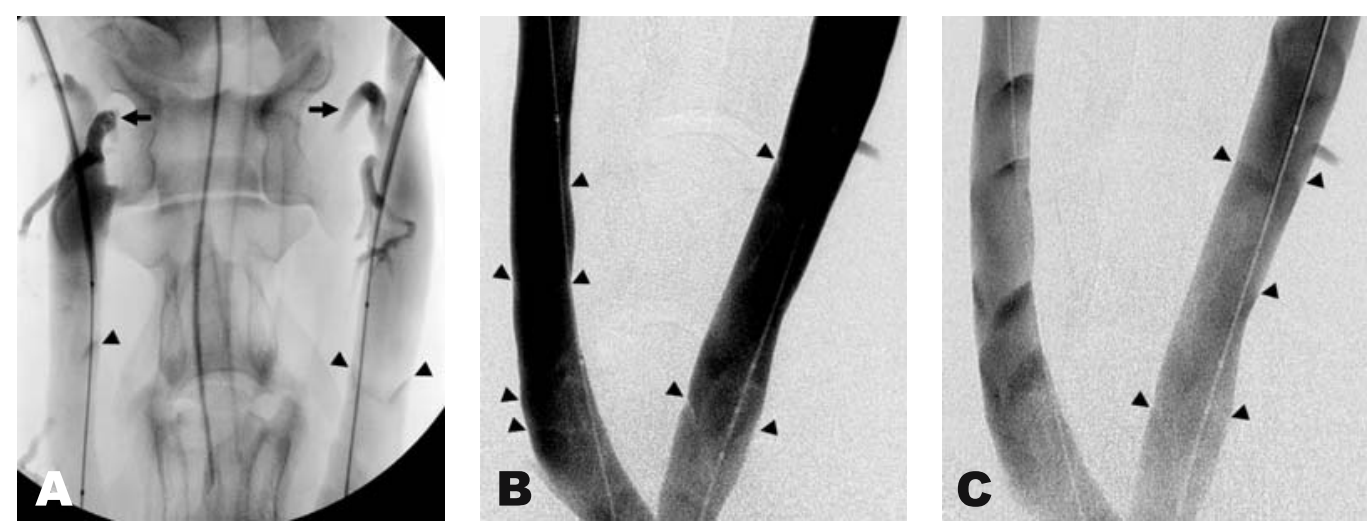

Figure 1 a-c. Ascending venograms of the jugular veins done in a titled position.

(a) Late phase venogram of the peripheral segment of the jugular vein demonstrates a monocusp valve on the right side (arrowhead) and bicuspid valve on the left side (arrowheads). There is filling of jugular vein tributaries and perforators vein (arrows).

(b) Early phase subtraction venograms of the middle and central segments of the jugular veins demonstrate valves as linear defects inside the vessel filled venous sinuses.

(c) Late phase subtraction venogram of the middle and central segments of the jugular veins demonstrates valves as residual filling in the valve cusps. Five bicuspid valves are seen on the right side and three on the left side (arrowheads). 

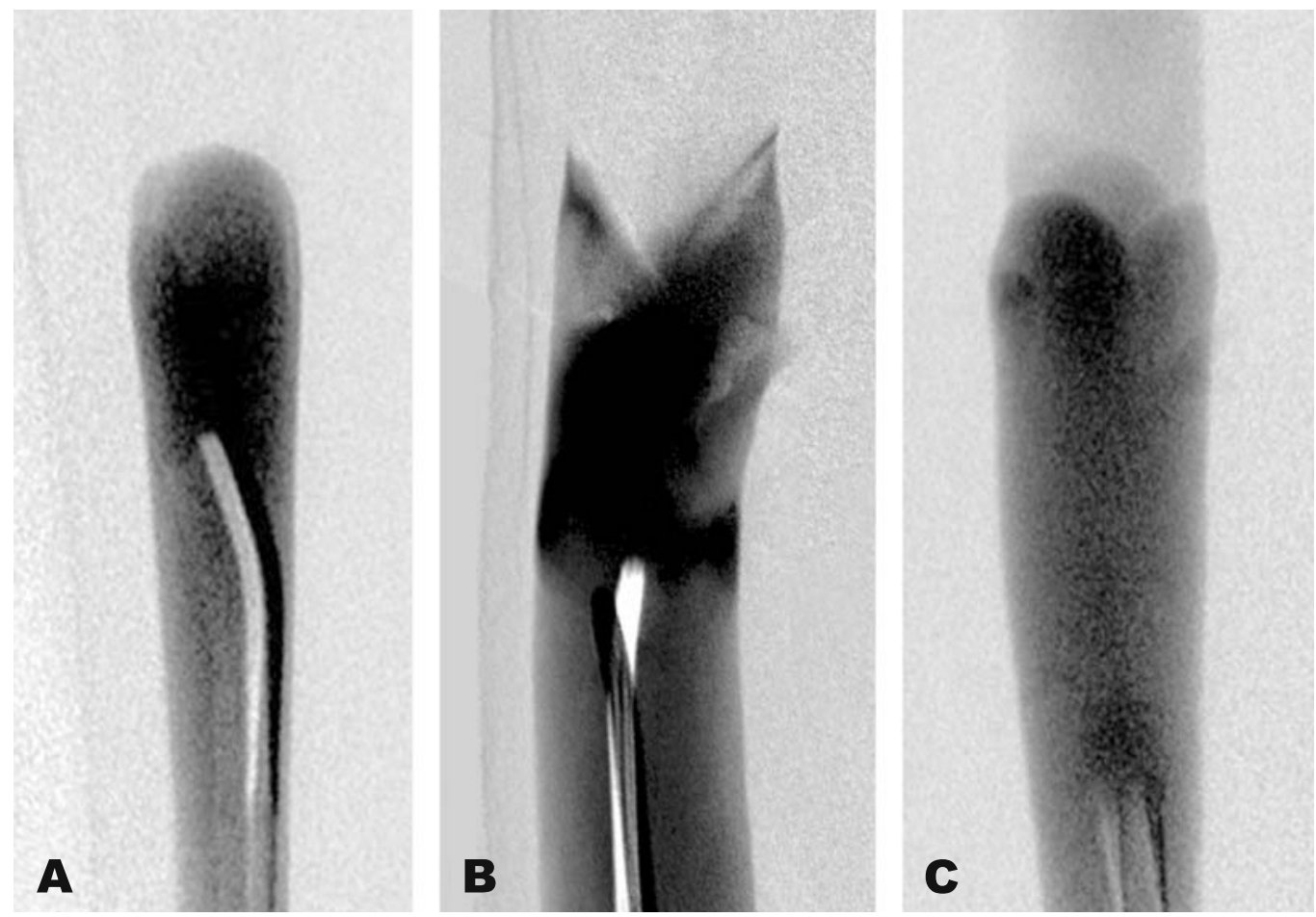

Figure 2 a-c. Descending venograms done in tilted position with injections central to valves demonstrate three types of valves. (a) Monocusp valve (b) Bicusps Valve (c) Tricusps valve.

when injection occurred below the most peripheral valve.

The specimens of $8 \mathrm{JVs}$ removed at the autopsies in four animals showed the same number, distribution and type of valves. All were bicuspid as seen on their venograms (Figure 3).

\section{Discussion}

Detailed knowledge of the ovine jugular vein anatomy and physiology is a prerequisite for proper use of sheep as teaching or an experimental model in interventional radiology. The ovine $\mathrm{JV}$, also called the external $\mathrm{JV}$, is the largest vein in the neck and drains most blood from the head and neck. The internal JV in sheep is small and often absent. ${ }^{11}$ The JV originates near the ventral border of the parotid gland at the angle of the mandible by the union of the external and internal maxillary veins. Traversing in the neck in the muscle groove, the JV accepts small tributary veins from thyroid, trachea, esophagus and muscles. Two axillary veins join the JV in its central segment at its entrance into the thorax. ${ }^{11}$ The right and left JVs then unite to form the superior vena cava. The JVs are thin-walled vessels and at surgery were found to have a mean diameter of approximately $9 \mathrm{~mm} .{ }^{8}$ During ascending venography in tilted position, the JVs distend and their mean diameter was $13.34 \pm 1.18$ in the presented series. The measurement of the maximal JV diameter during distention is important for selection of proper size of the valvular devices that we were testing. To prevent migration, 


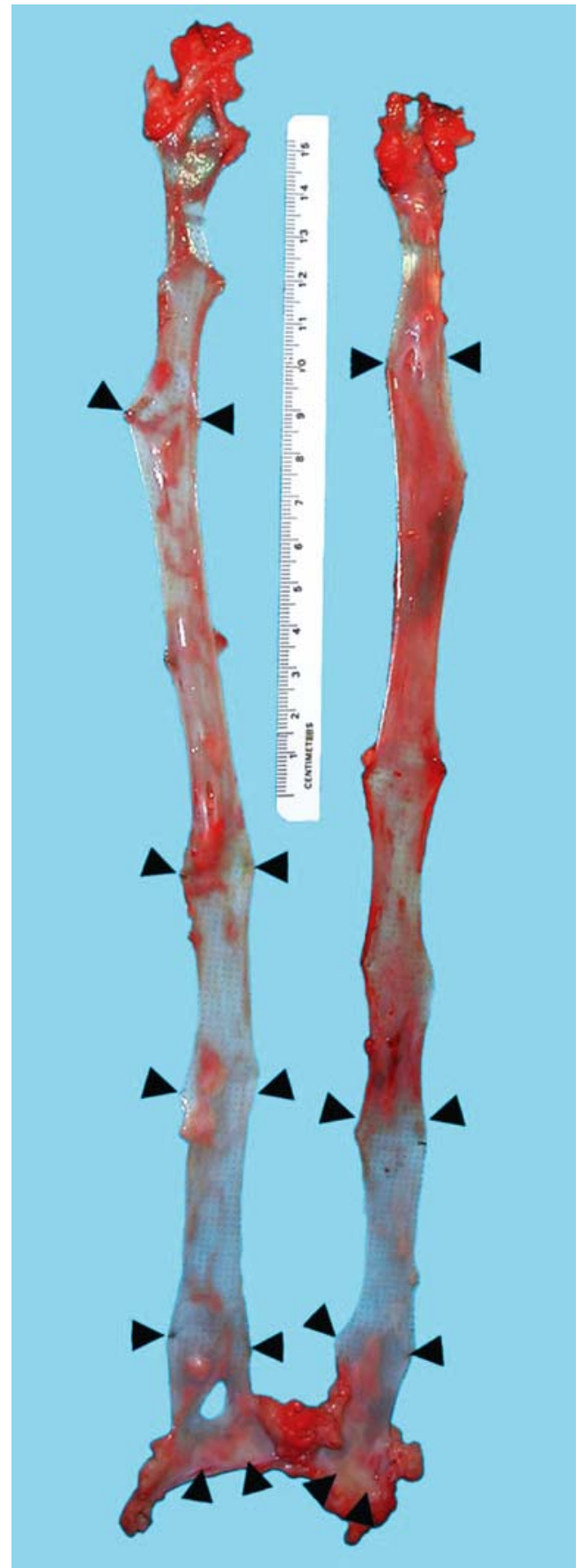

Figure 3. Longitudinally cut open specimen of both jugular veins $26 \mathrm{~cm}$ in length shows 5 bicuspid valves (arrow heads) on the right and four bicuspid valves on the left side (arrowheads).

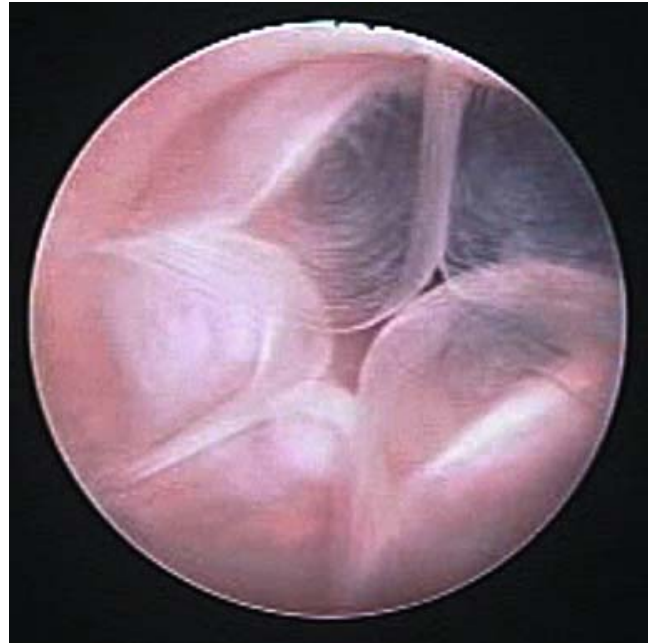

Figure 4. A pentacusps valve. Venoscopy shows five well functioning cusps.

the device should have a diameter about 15 to $20 \%$ larger than the vein diameter. ${ }^{3}$

The valves in veins are located peripherally to the entrance of large venous tributaries or junction of two veins of equal diameter. ${ }^{12}$ The valves close during increased central or hydrostatic pressure and prevent blood reflux and venous hypertension peripherally. Ascending venography demonstrated that the ovine JV contains one valve constant at its origin and two constant valves in its central segment at the entrance of two axillary veins. The number of valves in the middle segment was variable and ranged from zero to two. Venographic documentation of the presence and number of valves in the JV that compared well with the specimen studies is more accurate than their surgical identification. With valve identification by white semi-lunar lines formed by the attachment of the valve cusp to the vein wall, Jessup and Lane found only one to three valves in the jugular vein and in 3 of 32 veins $(9.4 \%)$ found no valves. ${ }^{8}$ The JV valves are mostly bicuspid, $96.3 \%$ in our series. JV valves are rarely monocuspid or tricuspid type. We found $2.3 \%$ and $1.4 \%$, respectively in our se- 
ries. However, valves with more than three cusps can also occur. In our previous experience with venoscopy of JV specimens, we found a (quintacusp) valve containing 5 well functioning cusps (Figure 4). In our series all $\mathrm{JV}$ valves evaluated by venography exhibited good function. All were competent and no venous reflux was seen during descending venography in tilted position. We consider venographic evaluation of valve competency more physiologic and accurate than the milking technique used by surgeons during open surgery. Using this technique, Jessup and Lane found that 18 of 32 JVs (56.3\%) in normal sheep had partially or completely incompetent valves. ${ }^{8}$

The ovine JV is a good model for evaluation of new percutaneously placed venous devices because of its similarities with the human femoral vein $(\mathrm{FV}) .^{3}$ These similarities include their diameters, number, and type of valves and function of their valves with increased central and hydrostatic pressures. The diameter of the ovine JV is around $13.34 \mathrm{~mm}$ in the tilted position and compares well with the diameter of the normal standing human FV of $10.0 \pm 0.21 \mathrm{~mm} .{ }^{13}$ The number and distribution of valves are also similar. The ovine JV contains a mean of 4.6 valves in a vein length of about $25 \mathrm{~cm}$ to $30 \mathrm{~cm}$. The human FV contains an average of 5 valves from the knee to the inguinal ligament including the constant valves at its central and peripheral end. ${ }^{14}$ Most of the valves in the ovine JV and the human FV are of bicuspid type and function similarly. They are open during relaxation and with muscle contraction. An increased central venous pressure causes the competent valves to close and prevent venous reflux and peripheral venous hypertension. In humans, the competent FV valves close with increase hydrostatic pressure in the upright position, and episodically pressure increases during deep breathing, straining and coughing. The function of JV valves in quadrupeds is to maintain the direction of the blood flow toward the heart and to protect the capillary beds of the head from the high venous pressure pulses caused by chest compressions and during eating and drinking with their heads down. ${ }^{15}$

Venographic studies are essential for both the evaluation of JV anatomy prior to prosthetic valve device placement and for following-up their function. Ascending venograms in the tilted position gives information about the JV size and position of their valves, particularly the central valve. As mentioned above, a prosthetic valve should be 15 to $20 \%$ larger than the JV diameter. The experimental prosthetic valves have been always placed across the central valve to replace its function. ${ }^{3,7,9}$ Because placement of one prosthetic valve will probably not solve chronic venous insufficiency, placement of two or more prosthetic valves will need to be evaluated in the ovine JV. Therefore, determination of position of other JV valves will be necessary. Descending venography can be done as a part of the preplacement evaluation, particularly if there is a question regarding the type and competency of the central valve. For follow-up studies, however, descending venography is the main procedure to evaluate valve competency. ${ }^{16,17}$ Ascending venography must also be done at that time to visualize the entire $\mathrm{JV}$ and evaluate any changes related to the prosthetic valve placement.

\section{Acknowledgements}

The authors thank Sheri Imai-Swiggart for her contribution. 


\section{References}

1. Gomez-Jorge J, Venbrux AC, MageeC. Percutaneous deployment of a valved bovine jugular vein in the swine venous system: a potential treatment for venous insufficiency. J Vasc Interv Radiol 2000; 11: 931-6.

2. Dalsing MC, Sawchuk AP, Lalka SG, Crikrit DF. An early experience with endovascular venous valve transplantation. J Vasc Surg 1996; 24: 903-5.

3. Pavcnik D, Uchida B, Timmermans HA, Corless CL, O'Hara M, Toyota N, et al. Percutaneous bioprosthetic venous valve: a long-term study in sheep. J Vasc Surg 2002; 35: 598-602.

4. de Borst GJ, Teijink JA, Patterson M, Quijano TC, Moll FL. A percutaneous approach to deep venous valve insufficiency with a new self-expanding venous frame valve. J Endovasc Ther 2003; 10: 341-9.

5. Teebken OE, Puschmann C, Apert T, Haverich A, Mertsching $\mathrm{H}$. Tissue-engineered bioprosthetic venous valve: a long-term study in sheep. Eur J Vasc Endovasc Surg 2003; 25: 305-12.

6. Pavcnik D, Uchida B, Timmermans HA, Keller FS, Rösch J. Square stent: a new self-expandable endoluminal device and its applications. Cardiovasc Intervent Radiol 2001; 24: 207-17.

7. Pavcnik D, Kaufman J, Uchida B, Correa L, Hiraki T, Kyu SC, et al. Second-generation percutaneous bioprosthetic valve: a short-term study in sheep. J Vasc Surg 2004; 40: 1223-7.

8. Jessup G, Lane RJ. Repair of incompetent venous valves: a new technique. J Vasc Surg 1988; 8: 56975

9. Pavcnik D, Kaufman JA, Uchida BT, Case B, Correa LO, Goktay AY, et al. Significance of spatial orientation of percutaneously placed bioprosthetic venous valves in an ovine model. J Vasc Interv Radiol 2005: 16: 1511-6.

10. Brountzos E, Pavcnik D, Uchida B, Corless C, Uchida BT, Nihsen ES, et al. Remodeling of suspended small intestinal submucosa venous valve: an experimental study in sheep to assess the host cells' origin. J Vasc Interv Radiol 2003; 14: 349-56.

11. May N. The Anatomy of the Sheep. 3r ed. Queensland, Australia: University of Queensland, Press; 1970. p. 133-4.

12. Gottlob R, May R. Venous valves. New York: Springer-Verlag Wien; 1986. p. 15-6.
13. van Bemmelen, PS: Evaluation of the patient with chronic venous insufficiency: old and emerging technologies. In Vascular Diseases. Surgical and Interventional Therapy, Churchill Livingstone Inc., New York 1994, 941-949.

14. Strandness DE: Applied Physiology of the Venous System. In Vascular Diseases. Surgical and Interventional Therapy, Churchill Livingstone Inc., New York 1994, 103-117.

15. Voorhees WD 3rd, Ralston SH, Babbs CF. Regional blood flow during cardiopulmonary resuscitation with abdominal counter pulsation in dogs. Am J Emerg Med 1984; 2: 123-8.

16. Pavcnik D, Yin Q, Uchida B, Park WK, Kim MD, Hoppe $\mathrm{H}$, et al: Percutaneous autologous venous valve transplantation: feasibility study in and ovine model. J Vasc Surg 2007; 46: 338-45.

17. Pavcnik D, Uchida B, Kaufman JA, Hinds M, Keller FS, Rösch J. Percutaneous management of chronic deep venous reflux: review of experimental work and early clinical experience with bioprosthetic valve. J Vasc Med 2008; 13: 75-84. 